\title{
TEACHING AND LEARNING INNOVATION IN PRESENT AND FUTURE OF HUMAN ANATOMY COURSE AT RSU
}

\author{
Dzintra Kažoka, Māra Pilmane \\ Department of Morphology, Institute of Anatomy and Anthropology, Riga \\ Stradinš University, Riga, Latvia
}

\begin{abstract}
With the emerging presence of IT based technologies in medical education, virtual anatomy has become a part of medical teaching, learning and healthcare. Building on the foundation of strong basic sciences, teaching and learning, Rìga Stradiņš University (RSU) offers its students the Anatomage Table. The Department of Morphology enabled new possibilities and allowed students to learn anatomy through a high-quality and interactive $3 \mathrm{D}$ tool.

The aim of this study was to investigate the usefulness of the Anatomage Table (Virtual Dissection Table) and overall satisfaction among students in the teaching and learning of Human Anatomy course at RSU.

The sample included Latvian and Foreign students and several tutors from the Human Anatomy course in the period of 2016-2017. This study was conducted in a real practical class context and under actual learning conditions. The Anatomage Table was used in two or three semester courses for the students of the Faculty of Medicine and the Faculty of Dentistry. In practical classes the learning, comparison and students satisfaction were explored. At the end of each practical class, the tutor performed a discussion session with the students' about increasing of their knowledge and success on the Human Anatomy study process.

The majority of students felt that the Anatomage Table had a positive role and it was an additional tool in terms of their learning. Students also noted several advantages of using the Anatomage Table in the medical teaching, learning and healthcare. The results indicated that learning Human Anatomy can be significantly impacted with virtual anatomy. The use of virtual dissection technology seems to have a promising role in future educational training.
\end{abstract}

Keywords: anatomy; virtual dissection; teaching; learning 


\section{INTRODUCTION}

Human Anatomy is one of the most important foundation disciplines and cornerstones in medical education. The level of knowledge in anatomy prepares the students for future in clinical disciplines [3]. In this time period the health sciences undergraduate can study the body using different strategies based on modern technology. The explosion of technologies during the last few decades has brought anatomical education into a new world $[10,16]$. With the continuous development of computer, mobile applications and progression the use of interactive technology in medical teaching and education is constantly evolving and increasing $[9,18]$. Today's learners are wired differently from the students of prior generations. A growing number of students now use electronic resources, access study materials on a smartphone and continue medical education online [13]. The teaching of Human Anatomy, like that of any course, requires the analysis to determine the teaching tools and approaches that best suit the learning process. Anatomy textbooks have become electronic and a lot of lectures are moving online. Companies with virtual anatomy products offer detailed, interactive anatomical images to complement textbooks and traditional cadaveric studies. For smaller Anatomy programmes the virtual anatomy products seek to raise the standard of health education by supplying new and innovative tools for exploring the human body. The Anatomage Table can be a foundation or a supplement to any Human Anatomy curriculum and teaching methods. The Anatomage Table is a life-size Virtual Dissection Table that displays gross anatomy models reconstructed from cadavers [6]. Anatomy can be presented in different planes and in the three-dimensional (3D) format. A combination of hardware and software allows the creation of case studies from computed tomography (CT) and magnetic resonance images (MRI) imported via DICOM files. The Anatomage Table is being used by hundreds of institutions around the world [17]. Building on the foundation of strong basic sciences, teaching and learning, Rīga Stradiņš University (RSU) offers its students the Anatomage Table. There is different information in the literature in regard to how the use of this technology is impacting students' learning.

The aim of this study was to investigate the usefulness and overall satisfaction among the students who utilized the Virtual Dissection Anatomage Table for the teaching and learning of the Human Anatomy course. 


\section{MATERIAL AND METHODS}

The Department of Morphology enabled new possibilities and allowed the students to learn anatomy through a high-quality and interactive, virtual 3D tool (Fig. 1). The sample included Latvian and Foreign students and several tutors from the Human Anatomy course in the period of 2016-2017. This study was conducted in a real practical class context and under actual learning conditions. The Anatomage Table was used in two or three semester courses for the students of the Faculty of Medicine and the Faculty of Dentistry. None of the participating students had ever worked with the Table. Practical classes involved the students gathered around the Table locating various anatomical or pathological structures as requested by the tutor (Fig. 2). The tutors served as facilitators guiding the students through the learning and developed the groups of students and the discussion questions based on previous experience and knowledge. In a practical class the following activities were used: viewing and dissecting the anatomy based on various body systems in different planes and images, and the analysis of the pathology case studies. Students were randomized between two methods. In practical classes the learning, comparison

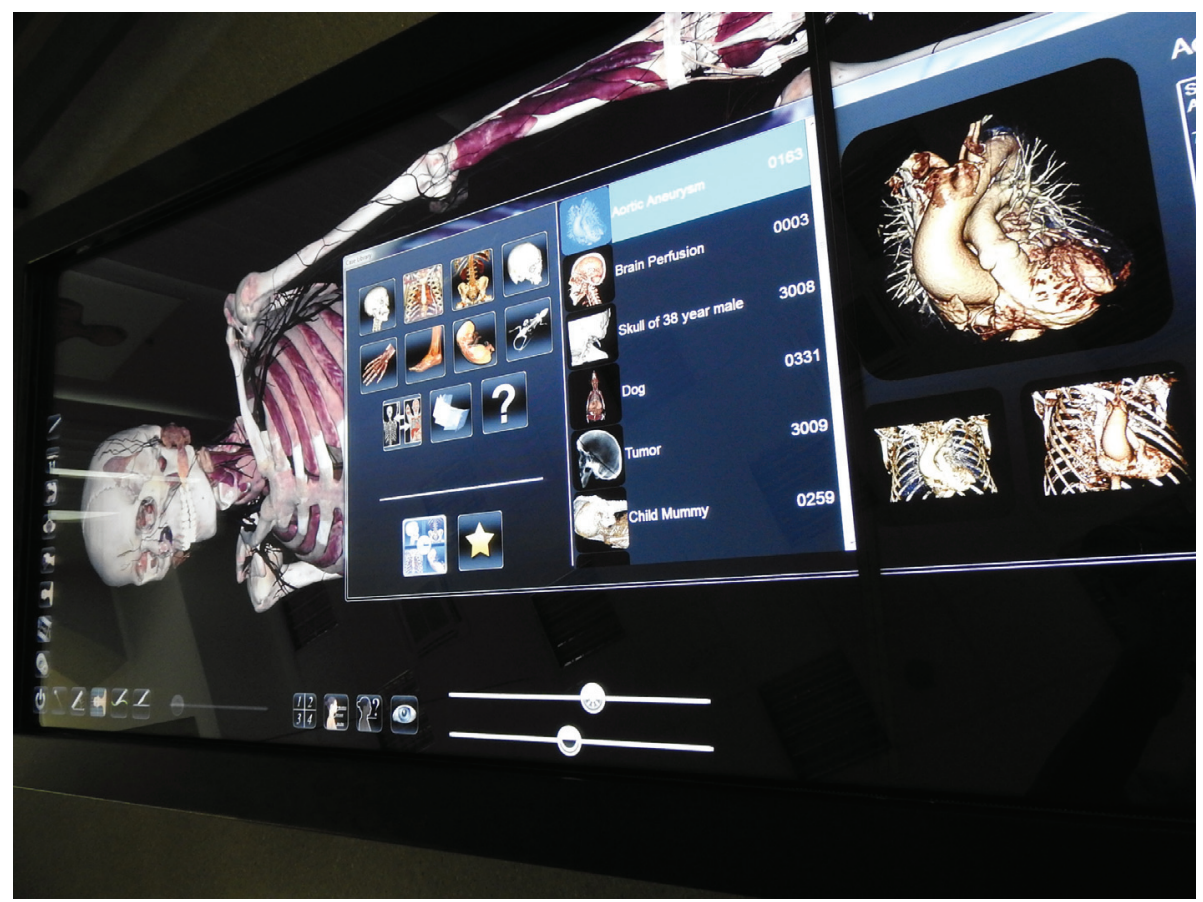

Figure 1. Some possibilities of the Virtual Dissection technology Anatomage. 
and students' satisfaction were explored. At the end of each practical class the tutor performed a discussion session with the students about increasing of their knowledge and success on the Human Anatomy study process.

\section{RESULTS}

Each class primarly utilized the male body on the Anatomage Table. In some classes the fully segmented bodies were used to identify the structures of differences. It offered tutors to take control of the Anatomage Table in their own practical classes, while demonstrating the material. The Anatomage Table

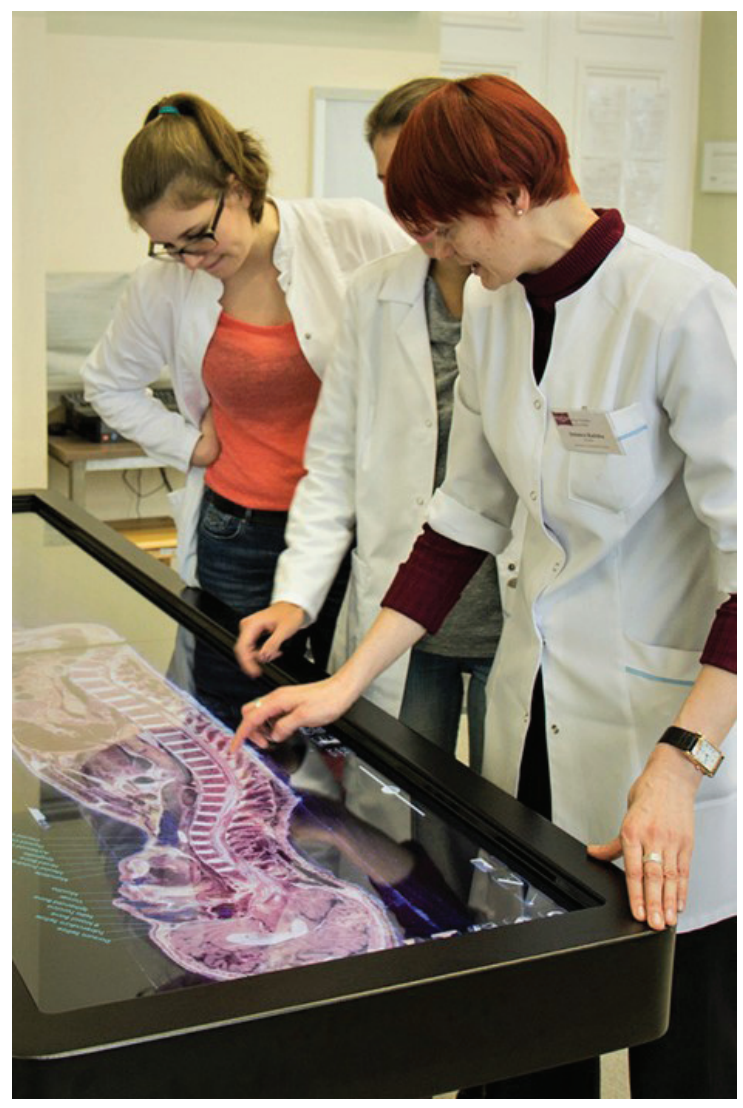

Figure 2. Students and the tutor using the Anatomage Table in the practical class. allowed students to more easily see different structures and layers, which were helpful for them as they had to know the details and the composition of systems. A lot of structures were labelled, so the students could easily quiz themselves or each other. Students were able to explore anything from bone to organs. The Table allowed the students to dissect hundreds of items, from humans to animals, from healthy organs to diseased ones. Certain regions were presented with more details in the high resolutions scans. The assessment of the discussions of students showed that the majority of them found that the Anatomage Table was an interesting and effective learning tool for developing their knowledge and skills, collaborative learning, using the anatomical language of images of dissections. The students who spent more time per class working on the Table had a more positive perception. The Table was an excellent supplement with other 
resources in teaching and learning of Human Anatomy. The results from the current study suggested that teaching Human Anatomy with the Anatomage Table offered some advantage on the questions requiring realtionships between structures. Special options increased the possibilities to imagine the rotation and positions of anatomical structures in different planes and from different perspectives. The Table helped students in the formation of a clearer representation of the structures, and contributed to improving learning and understanding of their verbal and visual knowledge. At the end of practical classes a lot of students mentioned topographical relationships between anatomical structures. The students started the description of the digital images with a very simple view of the anatomical structure. Step by step these were progressively complicated before being fully described and reported. Practically, all digital imaged provided sense and logic to some complex anatomical knowledge by combining the appropriate verbal description (given by the tutor) and visual information.

A way to improve student's learning is to change the method of teaching and allow students to take active participation. According to this, the students liked the ability to manipulate the virtual cadaver to better understand relational anatomy and cross sectional imaging.

Throughout the students activities, tutors shared what worked well, which did not, and what new ideas came from practical classes experience and exercises. This resulted in an increase in the use of the Anatomage Table from the beginning of the semesters to the end of them.

According to our results, the computer-assisted anatomy practical class was generally perceived by the students to be enjoyable. Students appreciated teaching and learning with the Anatomage Table.

\section{DISCUSSION}

The developmental intensity in technologies over the past few years has profoundly affected health care and medical education, and teaching Human Anatomy has been considerably changing during the last decade. We live in the time of the continuing evolution of computer-based multimedia technologies and there is much debate about the suitable methods of delivering anatomical knowledge. Our results support the findings that the best way to teach modern Anatomy is by combining multiple resources. In the field of human anatomy many medical tutors started the use of the three dimensional 
(3D) computers. The Anatomage Table is a unique technology that presents software with anatomy viewing and modelling tools, materials with virtual male and female body contents regional anatomy for teaching and education [11]. Several studies have mentioned the effectiveness and advantages of the Anatomage Table as a learning tool in the study of Human Anatomy [7, 12]. This tool can be used directly during lectures and practical classes. Each system or anatomical structure can be separated and reviewed individually. The Digital Anatomy Library users can explore unique case examples. Tutors can demonstrate different simple or complicated medical and surgical procedures to students. The Anatomage Table has several clinical applications. The Anatomage Table brings anatomical images to life, allowing students to dissect the body and move through layers of tissue to see the structures inside. The Anatomage Table can be utilized in a variety of ways to better demonstrate, learn and teach anatomy. Our findings also found that the possibilities of the Anatomage Table offer students to view and manipulate full-body male and female models through the use of three virtual cadavers. It is equipped with numerous pathological examples which can be used to evaluate and compare normal and abnormal anatomical structures. In practical classes students can rotate and manipulate structures from various views to identify anatomical structures and relationships between them.

Installed software also provides the ability for students and tutors to scroll through the entire body, to perform different activities along with quizzing and testing of the material. With the touch of a finger, students can expand the size of a body section to study its details and turn that body part in different directions, to study it from all angles. The Table allows the students or tutors to redo any cut or any mistake that could be detrimental when working directly on a cadaver. The Anatomage Table does not replace the cadaver experience, but rather broadens and deepens the information.

Any innovation in technology must be integrated with innovation in pedagogy [8]. The use of Virtual Dissection technology seems to have a promising role in future educational training. In future students and tutors together should determine what could be the most effective use of the Anatomage Table.

Several studies on teaching Human Anatomy have already compared new teaching methods to traditional ones [14]. We started to use technology to enhance medical education, but hope to see technology used not as a replacement for other learning tools, but actually as an additional tool to improve the educational process. 
A lot of digital images can be instructional tools for teaching Human Anatomy, but we recommend anatomy tutors to mix different teaching methods (new and traditional) in order to create the desired transfer of knowledge. Students need to learn not just anatomical structures and functions but also the relationships between surrounding structures. Our students directly benefited from the digital images during their practical classes. Finally, we suggest that the students and the tutors should use digital images and the animations.

The Anatomage Table can demonstrate a lot of details captured in the scan data that may be missed in educational plastic models or anatomical charts/ posters. The users can upload their own CT or MRI data of each system. The ability to upload and view any radiology image set (CT, MRI) expands the veriety of the Anatomage Table both inside and outside practical classes.

Students can explore parts of the human body that are difficult to view using other additional tools. With the size of the LCDs and displays in the practical classes, all students can view the image at the same time. Full body volume data of male and female virtual anatomy serve as a supplement to the existing cadaver curriculum. The students can review material with tutors before or after traditional dissection in practical classes. Users can select turning on or off independent volume structures or detect specific features.

The Anatomage Table's tools also allow to have self-review or labelled anatomical structures and the generation of images for research and presentations. Specific medical cases, if uploaded in CT, MRI or ultrasound, can be studied in full details. The tutors can help offer the high values of effective and Virtual Human Anatomy [5].

Several studies showed that this technology system is accepted by students in Medical, Dental Faculties, Universities or Health schools and can be used to support the curriculum and enhance students' skills in the visualization of anatomical relationships [15]. Some authors showed that 3D Anatomy has several disadvantages compared to traditional teaching [1]. Even when computerized improvements have developed a new area giving students a lot of elements to facilitate their approach to structures, the possibility of direct contact with tissues and anatomical elements cannot yet be replaced. The best possibility of all used resources is to complement one another [2,4].

This study described the learning, comparison and satisfaction of students in regard to the Anatomage Virtual Dissection Table. At RSU, the Anatomage Table is becoming an additional component of anatomical courses for medical studies. Based on true Human Anatomy, it offers a unique digital teaching plat- 
form and digital Anatomy offers various benefits. This system will not only assist students to learn anatomical details, but also provide the appreciation of $3 \mathrm{D}$ structures. It can help young students to learn the complex anatomy structure better and faster than only using traditional methods. The Anatomage Table improves understanding of complex anatomical structures, their special relationship and helps training of manual skills and hand-eye coordination. The use of Virtual Dissection technology seems to have a promising role in future educational training.

\section{REFERENCES}

1. Adamczyk C., Holzer M., Putz R., Fischer M.R. (2009). Student learning preferences and the impact of a multimedia learning tool in the dissection course at the University of Munich. Annals of Anatomy, 191, 4, 339-348. https://doi.org/10.1016/j.aanat.2009.03.003

2. Allen L.K., Bhattacharyya S., Wilson T.D. (2015). Development of an Interactive Anatomical Three-Dimensional Eye Model. Anatomical Sciences Education, May-June 8 (3), 275-282. https://doi.org/10.1002/ase.1487

3. Ang E.T., Sugand K., Hartman M., Seow C.S., Bay B.H., Abrahams P. (2012). Singapore's anatomical future: quo vadis? Anatomical Sciences Education, 5, 4, 234-240. https://doi.org/10.1002/ase.1286

4. Arora L. (2014). Anatomy teaching - a passion or a profession. Journal of Dental and Medical Sciences, 13, 9, 33-34. https://doi.org/10.9790/0853-13983334

5. Berman A.C. (2015). Good teaching is good teaching: a narrative review for effective medical educators. Anatomical Sciences Education, 8, 386-394. https://doi.org/10.1002/ase.1535

6. Bielby-Clarke K. (2015). Virtual dissection and human patient simulator use in Life Sciences. Proceedings of the Physiological Society, 34, SA057.

7. Brown J., Stonelake S., Anderson W., Abdulla M., Toms C., Farfus A., Wilton J. (2015). Medical student perception of anatomage - A 3D interactive anatomy dissection table. International Journal of Surgery, 23, 1, S17-S18. https://doi.org/10.1016/j.ijsu.2015.07.053

8. Chittaro L., Ranon R. (2007). Web3D technologies in learning, education and training: Motivations, issues, opportunities. Computers \& Education, 49, 3-18. https://doi.org/10.1016/j.compedu.2005.06.002

9. Custer T., Michael K. (2015). The Utilization of the Anatomage Virtual Dissection Table in the Education of Imaging Science Students. Journal of Tomography \& Simulation, 1, 102.

10. Fruhstorfer B.H., Palmer J., Brydges S., Abrahams P.H. (2011). The use of plastinated prosections for teaching anatomy - The view of medical students on 
the value of this learning resource. Clinical Anatomy, 24, 246-252. https://doi.org/10.1002/ca.21107

11. Fyfe G., Fyfe S., Dye D., Crabb H. (2013). Use of anatomage tables in a large first year core unit. Electric dreams, Proceeding ascilite, Sydney, 298-302.

12. Gross M., Masters C. (2017). Virtual Dissection: Using Active Learning with the Anatomage Table to Enhance Student Learning. The FASEB Journal, 31, 1, Suppl. Ib9.

13. Mavrych V. (2016). Modern Trends in Clinical Anatomy Teaching. MOJ Anat Physiol 2, 1, 00035. https://doi.org/10.15406/mojap.2016.02.00035

14. McMenamin P.G., Quayle M.R., McHenry C.R., Adams J.W. (2014). The production of anatomical teaching resources using three-dimensional (3D) printing technology. Anatomical Sciences Education, 7, 479-486. https://doi.org/10.1002/ase.1475

15. Narnaware R. (2017). Three Dimensional (3D) Virtual Human Cadaver, Anatomage: An effective teaching tool for Human Anatomy. The FASEB Journal, 31, 1, Suppl. Ib10.

16. Sugand K., Abrahams P.H., Khurana A. (2010). The Anatomy of Anatomy: A Review for Its Modernization. Anatomical Sciences Education, 3, 2, 83-93. https://doi.org/10.1002/ase.139

17. Thomson K., Williams A. (2017). Virtual Anatomy Teaching Aids. In: Forensic Science Education and Training. John Willey \& Sons Ltd., 137-145. https://doi.org/10.1002/9781118689196.ch10

18. Trelease R.B. (2016). From chalkboard, slides, and paper to e-learning: How computing technologies have transformed anatomical sciences education. Anatomical Sciences Education, 9, 6, 583-602. https://doi.org/10.1002/ase.1620

\section{Address for correspondence:}

Dzintra Kažoka

Rigga Stradiņš University, Institute of Anatomy and Anthropology

Kronvalda blv. 9, Riga, Latvia, LV-1010,

E-mail: Dzintra.Kazoka@rsu.lv

Tel. +37167060806 